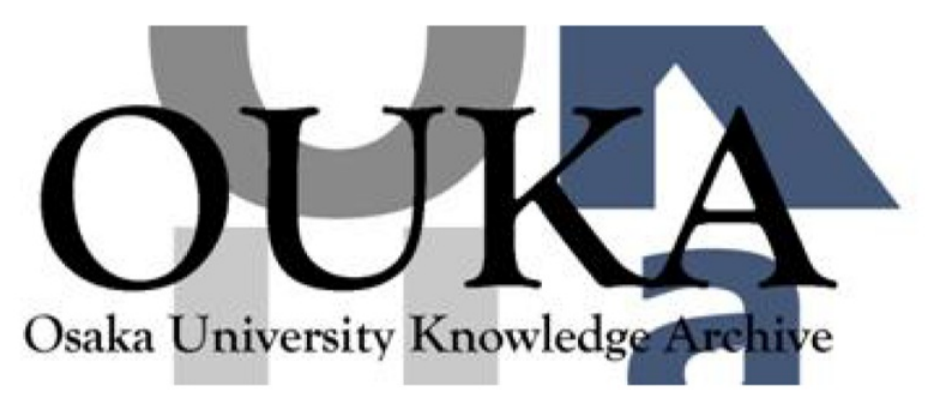

\begin{tabular}{|c|c|}
\hline Title & Dynamical chiral-symmetry breaking \\
\hline Author (s) & Higashijima, Kiyoshi \\
\hline Citation & $\begin{array}{l}\text { Physical review. Third series. D, Particles and } \\
\text { fields. } 29(6) \text { p. } 1228-p .1232\end{array}$ \\
\hline Issue Date & $1984-03$ \\
\hline oaire:version & VoR \\
\hline URL & https://hdl. handle. net/11094/3193 \\
\hline rights & $\begin{array}{l}\text { Higashijima, Kiyoshi, Physical review. Third } \\
\text { series. D, Particles and fields, 29, 6, 1228- } \\
\text { 1232, 1984-03. "Copyright } 1984 \text { by the American } \\
\text { Physical Society." }\end{array}$ \\
\hline Note & \\
\hline
\end{tabular}

Osaka University Knowledge Archive : OUKA

https://ir. Library. osaka-u. ac. jp/

0saka University 


\title{
Dynamical chiral-symmetry breaking
}

\author{
Kiyoshi Higashijima* \\ School of Physics and Astronomy, University of Minnesota, Minneapolis, Minnesota 55455
}

(Received 2 August 1983)

A solution of a quantum-chromodynamic Schwinger-Dyson equation for the quark propagator approximated in a way that is consistent with asymptotic freedom shows that chiral symmetry breaks down spontaneously.

\section{INTRODUCTION}

One of the important problems in particle physics is the question of whether or not quantum chromodynamics (QCD) really leads to the dynamical breakdown of chiral symmetry. ${ }^{1}$ Computer simulations in lattice gauge theory suggest that the chiral symmetry breaks down spontaneously $^{2}$ and that the range of force responsible for chiralsymmetry breaking is relatively short, independent of confinement. ${ }^{3}$ This result seems to be quite natural, since we expect intuitively a very small size for such a tightly bound state as a pion. In this paper, we derive the dynamical breakdown of chiral symmetry in continuum theory assuming that the relatively short-range force rather than the confining force is responsible for chiral-symmetry breakdown, as a complementary argument to the computer simulations.

Before going into technical details, let us recall a crude but basic idea about how the dynamical breakdown of chiral symmetry occurs in gauge theories. Consider a bound state of a pair of massless quark and antiquark. Because of the uncertainty principle, the energy of the ground state (pion) will be given by $E_{\pi} \approx p-g^{2} / r \approx p\left(1-g^{2}\right)$, where $p$ and $r$ denote the relative momentum and coordinate, respectively. In a fully relativistic formulation, this relation may be replaced by $E_{\pi}^{2} \approx p^{2}-g^{2} / r^{2} \approx p^{2}\left(1-g^{2}\right)$. When the gauge coupling constant exceeds order one, there will be a tachyon bound state, indicating instability of the vacuum. ${ }^{4}$ In order to cure this instability, the vacuum rearranges itself and gives mass to quarks so as to keep the pion massless. ${ }^{1}$ Hence, in the ladder approximation, the chiral symmetry is expected to break down spontaneously for coupling constants above a certain critical value.

The standard method to describe symmetry breaking in field theories is to evaluate the effective potential and to minimize it with respect to some local order parameters. It is quite difficult, however, to obtain an unambiguous effective potential when the order parameters are composite operators like $\langle\bar{q} q\rangle$ because of complications due to renormalizations. It is rather tractable to express the vacuum energy as a functional of a related nonlocal order parameter, the quark propagator itself. The extremum condition for the vacuum energy, in this case, leads to the Schwinger-Dyson (SD) equation. ${ }^{5}$ In this article, therefore, we solve the SD equation for the quark propagator.

The outline of the paper is as follows. In Sec. II we de- fine the improved SD equation. The asymptotic behavior of the quark propagator is found by solving the linearized SD equation. The current-quark mass is defined by the asymptotic behavior of the quark mass term. Numerical solutions of the nonlinear SD equation are presented in Sec. III. The relation between the current- and constituent-quark masses is also given in this section. Finally, Sec. IV consists of summary and some comments.

\section{IMPROVED SD EQUATION}

The SD equation is a nonlinear integral equation for the quark propagator with, in general, a complicated kernel. In QCD, however, there is a possibility of simplifying the SD equation. If the short-range force rather than the confining force is responsible for chiral-symmetry breaking in QCD as was suggested in Ref. 3, the kernel of the SD equation may be approximated by the one-gluon-exchange contribution since the coupling constant becomes smaller at short distances. With this ladderlike approximation, the SD equation in the Landau gauge assumes an especially simple form as ${ }^{6,7}$

$$
M\left(p^{2}\right)=\frac{1}{4 \pi^{2} i} \int d^{4} p^{\prime} \frac{\lambda}{-\left(p-p^{\prime}\right)^{2}-i \epsilon} \frac{M\left(p^{\prime 2}\right)}{M^{2}\left(p^{\prime 2}\right)-p^{\prime 2}},
$$

where

$$
\lambda \equiv 3 C_{2}(R) g^{2} / 4 \pi^{2}=(3 / \pi) C_{2}(R) \alpha_{S}
$$

and $C_{2}(R)$ denotes the Casimir invariant of the quark representation. The inverse quark propagator can be written as $S^{-1}(p)=-i\left[p-M\left(p^{2}\right)\right]$ in the Landau gauge. The above nonlinear SD equation, extensively studied by Maskawa and Nakajima, is known to have spontaneously broken solutions for $\lambda>1$ provided that an artificial ultraviolet cutoff is introduced in order to tame the violent short-distance singularity. ${ }^{8}$ In QCD, however, a natural short-distance cutoff is provided through the running coupling constant, so that it may be possible to obtain spontaneously broken solutions without introducing an artificial ultraviolet cutoff. In order to solve the SD equation numerically, we convert Eq. (1) into a differential equation. After introducing the Wick rotation and using the identity $\square\left(1 / x^{2}\right)=-4 \pi^{2} \delta^{(4)}(x)$, we obtain 9 


$$
\left[\frac{d^{2}}{d t^{2}}+4 \frac{d}{d t}+3\right] f(t)=-\lambda(t) \frac{f(t)}{1+f^{2}(t)}
$$

and boundary conditions

$$
\lim _{t \rightarrow \infty} e^{t}\left(\frac{d}{d t}+3\right) f(t)=0
$$

and

$$
\lim _{t \rightarrow \infty} e^{3 t}\left(\frac{d}{d t}+1\right) f(t)=0,
$$

where dimensionless variables $t$ and $f(t)$ are defined by $t \equiv \ln (p / \Lambda)$ and $M\left(p^{2}\right)=p f(t)$. (For later convenience, the renormalization invariant mass $\Lambda$ of QCD is chosen as an arbitrary mass scale.) We are now almost ready to "improve" our SD equation by identifying $\lambda(t)$ with the running coupling constant of QCD. If we do so, it will grow indefinitely at small- $t$ (large-distance) region and our approximation will break down. This behavior of the coupling constant is believed to lead to confinement. In this article, however, we are assuming that confinement is irrelevant for dynamical chiral-symmetry breaking. Hence, we throw away the confining force and define our nonconfining QCD-like model by (Fig. 1)

$$
\lambda(t)= \begin{cases}A / t & \left(t>t_{c}\right), \\ A / t_{c} & \left(t \leq t_{c}\right)\end{cases}
$$

where $A=6 C_{2}(R) /\left(11-2 n_{f} / 3\right)$ and $n_{f}$ denotes the number of flavors. From now on, we address ourselves to the above QCD-like model and solve the improved SD equation.

Asymptotic solutions of Eq. (2) are easily obtained. In the infrared region $(t \sim-\infty)$, the right-hand side of Eq. (2) can be neglected. Then, the boundary condition (4) forces us to choose a solution which behaves as

$$
f(t) \underset{t \rightarrow-\infty}{\sim}(M / \dot{\Lambda}) e^{-t}
$$

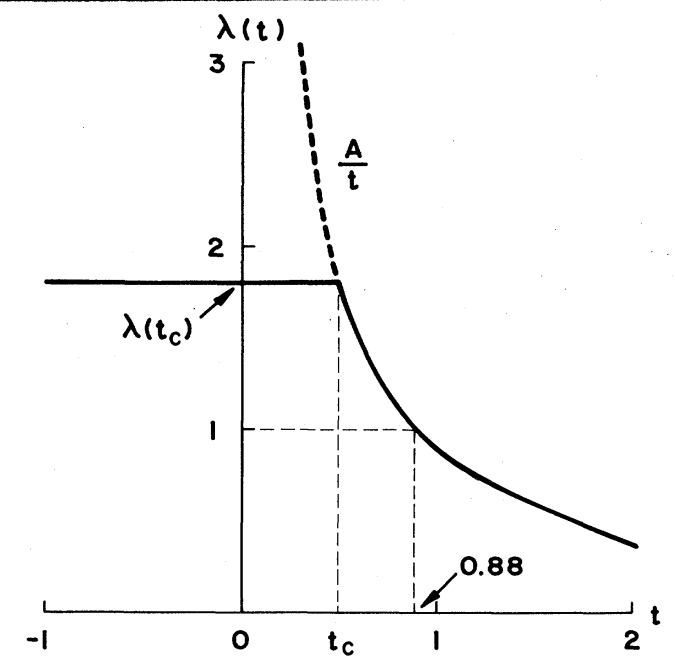

FIG. 1. Running coupling constant in QCD-like model for triplet quarks with $n_{f}=3$. where $M \equiv M(0)$ represents the mass term at low energy and may be called the constituent quark mass. In the ultraviolet region $(t \rightarrow \infty)$, the nonlinear term in Eq. (2) can be neglected. There, Eq. (2) reduces to the linear differential equation (confluent hypergeometric differential equation)

$$
\left\lfloor t \frac{d^{2}}{d t^{2}}+4 t \frac{d}{d t}+(A+3 t)\right] f(t)=0 .
$$

The asymptotic behavior of the solution is, in general, written as ${ }^{9,10}$

$$
f(t) \underset{t \rightarrow+\infty}{\sim}\left(\hat{m}_{R} / \Lambda\right) t^{-A / 2} e^{-t},
$$

where $\hat{m}_{R}$ denotes the multiplicatively renormalized (current-) quark mass. Since $\hat{m}_{R}$ appears in the renormalized Ward identity, it represents the explicit breaking of chiral symmetry. ${ }^{11,12}$

\section{NUMERICAL SOLUTION OF SD EQUATION}

Now our task is to solve the nonlinear SD equation numerically. In practice, $M$ has been given as an input, and the solution (6) at $t=-\infty$ has been continued to $t=+\infty$ by solving Eq. (2) to obtain the coefficient $\hat{m}_{R}$ defined by Eq. (8). By repeating this procedure for various values of $M=M(0)$, we obtain a relation of the constituent-quark mass $M$ and the current-quark mass $\hat{m}_{R}$. This relation between $M$ and $\hat{m}_{R}$ contains the infrared cutoff $t_{c}$ as a parameter. Dependence of the constituent-quark mass $M$ on the current-quark mass $\hat{m}_{R}$ is shown in Fig. 2 in the case of triplet quarks and $n_{f}=3$ for several values of $t_{c} \equiv \ln \left(P_{c} / \Lambda\right)$. For $t_{c} \lesssim 0.88 \quad\left(P_{c} / \Lambda \lesssim 2.2\right)$, the constituent-quark mass $M$ clearly remains nonvanishing in the chiral-symmetry limit $\hat{m}_{R}=0$, that is, the chiral symmetry is broken spontaneously. If we choose $t_{c}=0.4$ and $\Lambda=400 \mathrm{MeV}$, we obtain the constituent-quark mass $M=280$ (470) $\mathrm{MeV}$ for $\hat{m}_{R}=5$ (100) $\mathrm{MeV}$. Of course, we should not take these numbers too seriously, since the constituent-quark mass crucially depends on the choice of the infrared cutoff $t_{c}$. By plotting the values of $M$ at

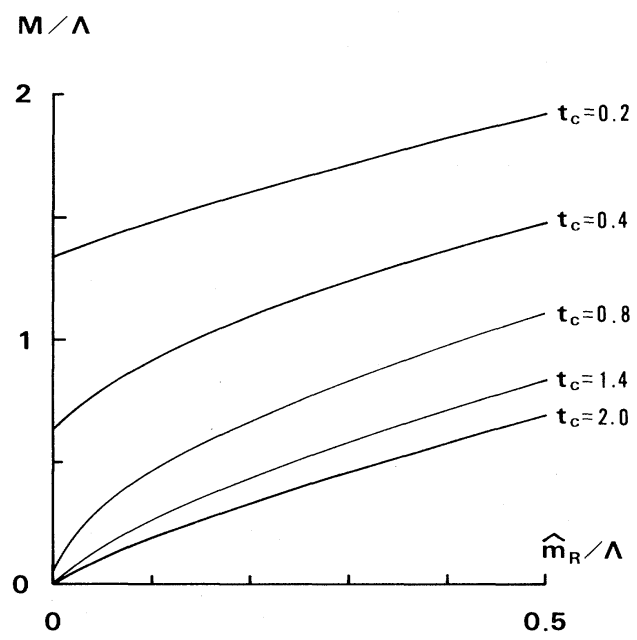

FIG. 2. Dependence of the constituent-quark mass $M$ on the current-quark mass $\hat{m}_{R}$ for triplet quarks with $n_{f}=3$. 
$\hat{m}_{R}=0$ for various values of $t_{c}$, we find how the dynamically generated mass $M$ depends on $t_{c}$ in the chiralsymmetry limit. Figure 3 shows the dependence of the constituent-quark mass $M$ on $P_{c} \equiv \Lambda \exp \left(t_{c}\right)$ when chiral symmetry is exact $\left(\hat{m}_{R}=0\right)$. Thus, in our QCD-like model, the chiral symmetry for triplet quarks is realized in the Nambu-Goldstone phase for $t_{c} \leq 0.88$ and in the Wigner phase for $t_{c}>0.88$. We have also studied this critical value of $t_{c}$ for other color representations of quarks. We found critical values $t_{c} \approx 1.9,1.8$, and 3.5 for $\underline{6}-, \underline{8}-$, and 10 -dimensional quark representations, respectively.

It is amusing to compare these numerical results with our intuitive picture of chiral-symmetry breakdown. Since $C_{2}(R)=\frac{4}{3}, \frac{10}{3}, 3$, and 6 for $\underline{3}^{-}, \underline{6}_{-}^{-}, \underline{8}^{-}$, and $\underline{10}-$ dimensional color representations, $A$ in Eq. (5) is given by $\frac{8}{9}, \frac{20}{9}, 2$, and 4 for these representations, respectively, when $n_{f}=3$. Then, coupling constants $\lambda\left(t_{c}\right) \equiv A / t_{c}$ evaluated at these critical values of $t_{c}$ are equal to one, independent of quark representations.

As seen from Fig. 1, if we take $t_{c}$ smaller than 0.88 , the critical value determined by $\lambda\left(t_{c}\right)=1$, we are including the effect of the relatively strong coupling region. In this case, the attractive force between $q \bar{q}$ may be strong enough to make a bound state (pion). Since a bound state of massless particles is necessarily a tachyon in nonconfining theories like our model, the chiral-symmetric vacuum becomes unstable. In order to cure this instability, the quark acquires a dynamical mass $M$ and the bound state (pion) becomes a massless Nambu-Goldstone boson. In fact, it is not difficult to derive a relation between the BetheSalpeter amplitude $\chi(p ; P)$ of the pion and the quark propagator $S(p) \equiv i z\left(p^{2}\right)\left[p-M\left(p^{2}\right)\right]^{-1}$ in the chiral-symmetry limit $\left(\hat{m}_{R}=0\right)$, namely, ${ }^{7,13}$

$$
F_{\pi} \chi(p ; 0)=\frac{2 z\left(p^{2}\right) M\left(p^{2}\right)}{p^{2}-M^{2}\left(p^{2}\right)} \gamma_{5},
$$

where the pion decay constant $F$ is defined by

$$
i P^{\mu} F_{\pi} \equiv Z_{2}{ }^{-1} \int \frac{d^{4} p}{(2 \pi)^{4}} \operatorname{Tr}\left[\gamma^{\mu} \gamma_{5} \chi(p ; P)\right]
$$

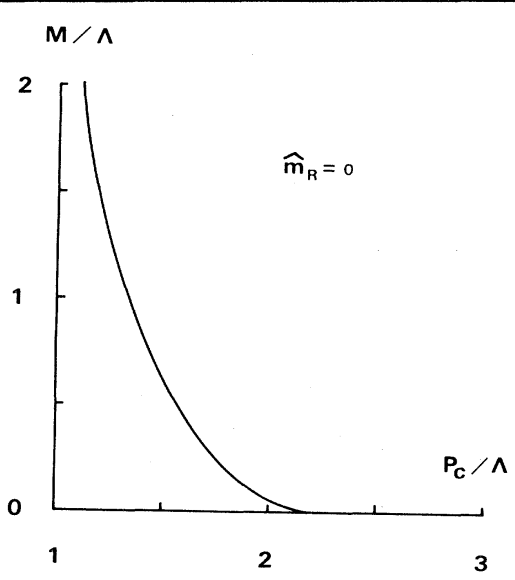

FIG. 3. Dependence of the constituent-quark mass $M$ on $p_{c}=\Lambda \exp \left(t_{c}\right)$ when chiral symmetry is exact, i.e., $\hat{m}_{R}=0$. with $Z_{2}$ being the wave-function renormalization constant of the quark. Equation (9) tells us that nonvanishing $M\left(p^{2}\right)$ implies the existence of the massless NambuGoldstone boson.

On the other hand, if we take $t_{c}$ larger than the critical value determined by $\lambda\left(t_{c}\right)=1$, the attractive force between $q \bar{q}$ is too weak to make a bound state. Therefore, the chiral-symmetric vacuum is stable and the quark remains massless in this case.

In summary, a criterion for dynamical chiral-symmetry breakdown is given by $\lambda\left(t_{c}\right) \geq 1$, in accordance with our intuitive picture discussed in the Introduction.

\section{SUMMARY AND DISCUSSION}

We may summarize our results as follows. We have defined our QCD-like model by throwing away the strongcoupling region where confinement takes place, nevertheless, we found that chiral symmetry breaks down spontaneously as far as the effect of the intermediate coupling region is incorporated, namely, as far as the condition $\lambda\left(t_{c}\right) \gtrsim 1$ is satisfied. Since in confining theories like QCD $\lambda(t)$ increases indefinitely as $t$ tends to minus infinity, there is certainly an intermediate coupling region where $\lambda \geq 1$. Therefore, we may conclude that chiral symmetry has to break down spontaneously in QCD. In other words, confinement is a sufficient but not a necessary condition for chiral-symmetry breaking.

Now some comments are in order.

(i) Consistency with the renormalization group (RG). Since we have discussed a nonperturbative effect by solving the SD equation, comparison of our results with those obtained by the RG equation is important for a check of reliability. The $\mathrm{RG}$ equation for the quark propagator $S(p)=i z\left(p^{2}\right)\left[p-M\left(p^{2}\right)\right]^{-1}$ gives the asymptotic behaviors of $z$ and $M$ :

$$
\begin{aligned}
& z\left(p^{2}\right) \underset{\left|p^{2}\right| \rightarrow \infty}{\rightarrow} 1, \\
& M\left(p^{2}\right) \underset{\left|p^{2}\right| \rightarrow \infty}{\rightarrow} \hat{m}_{R}\left(\frac{1}{2} \ln \frac{-p^{2}}{\Lambda^{2}}\right)^{-A / 2},
\end{aligned}
$$

where

$$
\hat{m}_{R} \equiv m_{R}\left[\frac{1}{2} \ln \frac{\mu^{2}}{\Lambda^{2}}\right]^{A / 2}
$$

denotes the renormalization point $(\mu)$ independent current-quark mass. Equation (8) coincides with Eq. (11). Therefore, the behavior of the quark mass term due to the explicit chiral-symmetry breaking $\left(\hat{m}_{R}\right)$ is consistent with the RG.

When chiral symmetry is exact $\left(\hat{m}_{R}=0\right), \boldsymbol{M}\left(p^{2}\right)$ vanishes identically in the chiral-symmetric phase. If the chiral symmetry is broken spontaneously, the quark mass term $M\left(p^{2}\right)$ no longer vanishes but is generated dynamically. The behavior of this dynamically generated mass term has been discussed by Politzer using the operatorproduct expansion: ${ }^{14}$

$$
M\left(p^{2}\right)=\frac{\bar{g}^{2}\left(p^{2}\right)}{3 p^{2}} \phi\left[\ln \frac{-p^{2}}{\Lambda^{2}}\right]^{A / 2},
$$


where

$$
\phi \equiv \frac{\left\langle 0\left|(\bar{q} q)_{R}\right| 0\right\rangle}{\left(\ln \mu^{2} / \Lambda^{2}\right)^{A / 2}}
$$

is the renormalization-point-independent vacuum expectation value of $\bar{q} q$. Because of $1 / p^{2}$ in Eq. (12), the mass term due to the spontaneous breaking of chiral symmetry decreases rapidly at high energy, and does not introduce a new mass counterterm. Since the linearized SD equation is a second-order differential equation, it has two fundamental solutions. Therefore, the asymptotic behavior of the solution is given by another (nonleading) solution: ${ }^{10,11}$

$$
M\left(p^{2}\right) \underset{\left|p^{2}\right| \rightarrow \infty}{\sim} C \frac{\Lambda^{3}}{p^{2}}\left[\frac{1}{2} \ln \frac{-p^{2}}{\Lambda^{2}}\right]^{A / 2},
$$

when $\hat{m}_{R}$ vanishes. By comparing Eqs. (12) and (13), we notice a discrepancy in the power of logarithm although $1 / p^{2}$ is the same. This is caused by our simple introduction of the running constant in Eq. (1): $g^{2} \rightarrow \bar{g}^{2}\left(p^{\prime 2}\right)$. Instead, we can recover the consistency with the RG by replacing $g^{2}$ in Eq. (1) with ${ }^{15}$

$$
\bar{g}^{2}\left(p_{>}^{2}\right) \equiv \theta\left(p-p^{\prime}\right) \bar{g}^{2}\left(p^{2}\right)+\theta\left(p^{\prime}-p\right) \bar{g}^{2}\left(p^{\prime 2}\right) .
$$

With this change, Eq. (2) will be replaced by

$$
\ddot{f}+4 \dot{f}+3 f-U(f+\dot{f})=-\left(\lambda-\frac{1}{2} \dot{\lambda}\right) \frac{f}{1+f^{2}},
$$

where

$$
U \equiv(\ddot{\lambda}-2 \dot{\lambda}) /(\dot{\lambda}-2 \lambda)
$$

Now the asymptotic solution of Eq. (15) is given by

$$
\begin{aligned}
M\left(p^{2}\right)= & \hat{m}_{R}\left[\frac{1}{2} \ln \frac{-p^{2}}{\Lambda^{2}}\right]^{-A / 2} \\
& +c \bar{g}^{2}\left(p^{2}\right) \frac{\Lambda^{3}}{p^{2}}\left[\frac{1}{2} \ln \frac{-p^{2}}{\Lambda^{2}}\right]^{A / 2}
\end{aligned}
$$

which is completely consistent with the RG. ${ }^{16}$ Since Eq. (17) is consistent with the RG, in principle, we can find a relation of $\hat{m}_{R}, M$, and $\phi \equiv \frac{1}{3} c \Lambda^{3}$ as we did in the previous section. In this paper, however, we used the simplest im- provement because we found no qualitative difference in our conclusion.

(ii) Choice of gauge. Throughout this paper we used the Landau gauge. Although the expressions (11) and (12) are derived in the Landau gauge, we note that these expressions are independent of the choice of gauge parameter as far as the leading behaviors are concerned, since the Landau gauge is a fixed point ${ }^{17}$ in the RG. On the other hand, it seems difficult to reproduce these expressions to the solutions of SD equation in the ladder approximation with a gauge other than the Landau gauge. ${ }^{18}$ Therefore, the Landau gauge seems to be the favorite gauge in the ladder approximation, in the sense that the correct asymptotic behaviors are reproduced in this special gauge.

(iii) Effect of the deconfining phase transition. At finite temperature, it is important to know the effect of the deconfining phase transition on the spontaneous breakdown of chiral symmetry. As we found, confinement is not necessary for the dynamical chiral-symmetry breaking if there is an intermediate coupling region. That is, the temperature of chiral-symmetry restoration $\left(T_{F}\right)$ is higher than the temperature of deconfining phase transition $\left(T_{c}\right)$. At the deconfining phase transition, the long-range confining part of the potential disappears, whereas the shortrange Coulomb potential remains as before. What is not clear is the fate of the potential in the intermediate coupling region. If this intermediate coupling region, where $\lambda \gtrsim 1$, survives the deconfining phase transition, the leftover unconfining force is still sufficient to break chiral symmetry, then $T_{F}$ is significantly higher than $T_{c}$. If the force in the intermediate coupling region disappears together with the confining force at the deconfining phase transition, the leftover short-range force is not strong enough to break chiral symmetry, then $T_{F}$ will be closed to $T_{c}$ in this case. Recent Monte Carlo simulation ${ }^{19}$ in lattice gauge theory suggests the latter possibility.

\section{ACKNOWLEDGMENTS}

The author is grateful to S. Gasiorowicz, K. Nishijima, and T. Eguchi for useful comments. He also wishes to express his sincere thanks to members of the Department of Physics and Astronomy for their kind hospitality shown to him at the University of Minnesota.
*On leave of absence from The University of Tokyo, Tokyo, Japan.

${ }^{1}$ Y. Nambu and G. Jona-Lasinio, Phys. Rev. 122, 345 (1961).

${ }^{2}$ H. Hamber and G. Parisi, Phys. Rev. Lett. 47, 1792 (1981); E. Marinari, G. Parisi, and C. Rebbi, ibid. 47, 1795 (1981).

${ }^{3}$ J. Kogut, M. Stone, H. W. Wyld, J. Shigemitsu, S. H. Shenker, and D. K. Sinclair, Phys. Rev. Lett. 48, 1140 (1982).

${ }^{4}$ A. Casher, Phys. Lett. $\underline{83 B}, 395$ (1979).

5J. Cornwall, R. Jackiw, and E. Tomboulis, Phys. Rev. D 10, 2428 (1974).

6For earlier references, see H. Pagels, Phys. Rep. 16C, 219 (1975).

${ }^{7}$ K. Higashijima and A. Nishimura, Nucl. Phys. B113, 173 (1976).
${ }^{8}$ T. Maskawa and H. Nakajima, Prog. Theor. Phys. 2ㅡ, 1326 (1974); $\underline{54}, 860$ (1975).

${ }^{9} \mathrm{We}$ will discuss the behavior of $M\left(p^{2}\right)$ only in the spacelike region. For the timelike region, see R. Fukuda and T. Kugo, Nucl. Phys. B117, 250 (1976).

${ }^{10}$ K. Lane, Phys. Rev. D 10, 2605 (1974).

${ }^{11}$ H. Pagels, Phys. Rev. D 19, 3080 (1979).

${ }^{12}$ S. Weinberg, Phys. Rev. D $\underline{8}, 455$ (1973).

${ }^{13}$ R. Delbourgo and M. D. Scadron, J. Phys. G $\underline{5}, 1621$ (1979).

${ }^{14}$ H. D. Politzer, Nucl. Phys. B117, 397 (1976).

${ }^{15}$ K. Higashijima, Phys. Lett. $\underline{124 B}, 257$ (1983).

${ }^{16}$ It has been shown that the leading UV behavior of $M\left(p^{2}\right)$ is given by Eq. (7), see Ref. 10; S. H. Tye, E. Tomboulis, and E. C. Poggio, Phys. Rev. D 11, 2839 (1975); J. M. Cornwall and 
R. C. Shellard, ibid. 18, 1216 (1978).

${ }^{17}$ For example, E. de Rafael, in Lecture Notes in Physics, Vol. 118 , edited by J. L. Alonso and R. Tarrach (Springer, Berlin, 1980).

${ }^{18}$ In another gauge, we have to introduce a vertex correction consistent with the Ward identity $Z_{1}=Z_{2}$. In this connection, see J. E. King, Phys. Rev. D 27, 1821 (1983).

${ }^{19}$ J. Kogut, M. Stone, H. W. Wyld, W. R. Gibbs, J. Shigemitsu, S. H. Shenker, and D. K. Sinclair, Phys. Rev. Lett. 50, 393 (1983). 